\title{
Vitamin A Deprivation Affects the Progression of the Spermatogenic Wave and Initial Formation of the Blood-testis Barrier, Resulting in Irreversible Testicular Degeneration in Mice
}

\author{
Masataka CHIHARA ${ }^{1)}$, Saori OTSUKA ${ }^{1}$, Osamu ICHII') and Yasuhiro KON ${ }^{1)}$ \\ 1)Laboratory of Anatomy, Department of Biomedical Sciences, Graduate School of Veterinary Medicine, Hokkaido \\ University, Sapporo 060-0818, Japan
}

\begin{abstract}
The blood testis-barrier (BTB) is essential for maintaining homeostasis in the seminiferous epithelium. Although many studies have reported that vitamin $\mathrm{A}(\mathrm{VA})$ is required for the maintenance of spermatogenesis, the relationships between the BTB, spermatogenesis and VA have not been elucidated. In this study, we analyzed BTB assembly and spermatogenesis in the testes of mice fed the VA-deficient (VAD) diet from the prepubertal period to adulthood. During the prepubertal period, no changes were observed in the initiation and progression of the first spermatogenic wave in mice fed the VAD diet. However, the numbers of preleptotene/leptotene spermatocytes derived from the second spermatogenic wave onwards were decreased, and initial BTB formation was also delayed, as evidenced by the decreased expression of mRNAs encoding BTB components and VA signaling molecules. From 60 days postpartum, mice fed the VAD diet exhibited apoptosis of germ cells, arrest of meiosis, disruption of the BTB, and dramatically decreased testis size. Furthermore, vacuolization and calcification were observed in the seminiferous epithelium of adult mice fed the VAD diet. Re-initiation of spermatogenesis by VA replenishment in adult mice fed the VAD diet rescued BTB assembly after when the second spermatogenic wave initiated from the arrested spermatogonia reached the preleptotene/leptotene spermatocytes. These results suggested that BTB integrity was regulated by VA metabolism with meiotic progression and that the impermeable BTB was required for persistent spermatogenesis rather than meiotic initiation. In conclusion, consumption of the VAD diet led to critical defects in spermatogenesis progression and altered the dynamics of BTB assembly.
\end{abstract}

Key words: Blood-testis barrier, Spermatogenesis, Vitamin A

(J. Reprod. Dev. 59: 525-535, 2013)

M ammalian spermatogenesis is a complex but highly orchestrated process that is generally divided into 3 phases: mitotic proliferation of stem spermatogonia, meiotic differentiation of spermatocytes, and transformation of spermatids into spermatozoa. Primary spermatocytes undergo spermatogenesis through the following 6 stages: preleptotene, leptotene, pachytene, zygotene, diplotene, and diakinesis; this is followed by entry into metaphase I. The first wave of spermatogenesis, meiosis initiation, occurs after birth and continues cyclically throughout adulthood (Fig. 1A). In mice, the spermatogenic cycle is divided into distinct stages (I-XII) with a stage-specific set of germ cells in the seminiferous tubules [1]. Four cycles of 8.62 days each (for a total of 34.48 days) are required to progress from spermatogonia to spermatozoa [2].

Male germ cell differentiation relies largely on Sertoli cells for structural and nutritional support. Sertoli cells maintain the integrity of spermatogenesis by forming the blood-testis barrier (BTB) [3, 4]. In adult animals, the BTB divides the seminiferous epithelium into adluminal and basal compartments, thereby establishing a suitable milieu for germ cell development. In several species, the BTB is

Received: May 20, 2013

Accepted: July 9, 2013

Published online in J-STAGE: August 12, 2013

(C)2013 by the Society for Reproduction and Development

Correspondence: Y Kon (E-mail: y-kon@vetmed.hokudai.ac.jp) initially established during the prepubertal period when the majority of germ cells derived from the first spermatogenic wave reach the early-pachytene stage [5-7]. In rats, BTB assembly does not occur synchronously along the length of the seminiferous tubules, despite the emergence of pachytene spermatocytes [6]. Hence, initial assembly of the BTB may be regulated by cooperation between the action of pachytene spermatocytes derived from the first spermatogenic wave and other unknown factors. After BTB assembly, preleptotene/ leptotene spermatocytes derived from the second wave onward need to migrate across the BTB from the basal compartment to the adluminal compartment for further development during spermatogenesis (Fig. 1A). In rodents, germ cell migration across the BTB occurs from late-stage VIII to early-stage IX [8].

Interestingly, to accommodate such germ cell migration, the expression and localization of tight junction (TJ) proteins that constitute the BTB, such as occludin (OCLN), claudins (CLDNs), and zonula occludens (ZOs), are altered in a seminiferous epithelium cycle-dependent manner $[9,10]$. Furthermore, although the expression levels of TJ transcripts and proteins in the testis increase with age in the prepubertal period and decrease with sexual maturation, these proteins consistently localize to the BTB throughout the reproductive period [9, 11-13]. Recently, some researchers have reported that Ocln- and Cldn11-null mice are sterile $[14,15]$ and that irreversible BTB disruption by adjudin inhibits differentiation from spermatogonia to spermatocytes [16]. Altered CLDN11 expression and localization 
A

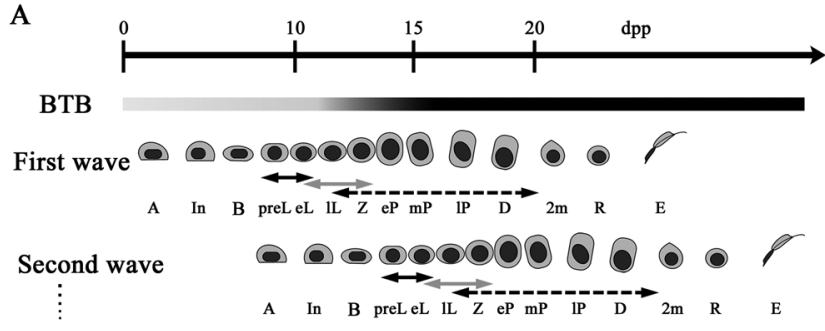

B

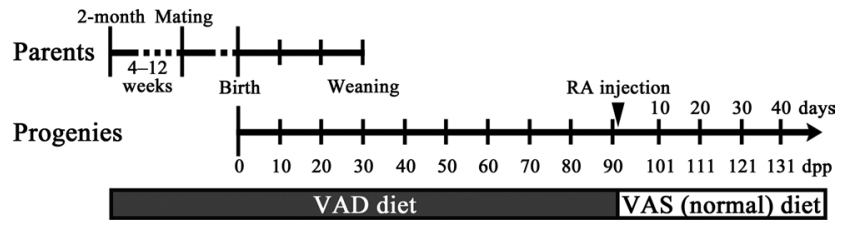

Fig. 1. Illustration of spermatogenic progression and BTB assembly during the prepubertal period and VAD diet feeding schedule. A: Time-course progression of spermatogenic waves with BTB assembly in prepubertal mice. The degree of BTB assembly and impermeability are indicated by the darkness of the shading at each time point. A, type A spermatogonia; In, intermediate spermatogonia; B, type B spermatogonia; preL, preleptotene spermatocytes; eL, early leptotene spermatocytes; IL, late leptotene spermatocytes; eP, early pachytene spermatocytes; $\mathrm{mP}$, mid pachytene spermatocytes; $1 \mathrm{P}$, late pachytene spermatocytes; $\mathrm{Z}$, zygotene spermatocytes; $\mathrm{D}$, diplotene spermatocytes; $2 \mathrm{~m}$, secondary spermatocytes; R, round spermatids; E, elongating spermatids. Solid black arrows, gray arrows, and black dashed arrows represent STRA8-, DMC1-, and SYCP3-positive spermatocytes, respectively. B: VAD diet feeding schedule used in the present study. Parental mice were fed the VAD diet from 4-12 weeks prior to mating, and male pups received the same diet until 90 dpp. At 91 dpp, mice fed the VAD diet were injected with RA and were then placed on a normal VAS diet.

at the BTB are also associated with the development of human testicular intraepithelial neoplasia [17]. These reports indicate that maintenance of BTB integrity is essential for normal spermatogenesis.

In mammals, retinoic acid (RA), the active derivative of vitamin A, has been shown to be an essential inducer of meiosis in both sexes [18]. Indeed, testes of rats and mice fed a vitamin A-deficient (VAD) diet show spermatogenic arrest at spermatogonia [19, 20]. RA administration and dietary retinoid replenishment in rodents fed the VAD diet result in the re-initiation of spermatogenesis, starting from the remaining spermatogonia, and synchronized spermatogenesis [21]. RA acts through binding to nuclear RA receptors (RARs) and retinoid X receptors (RXRs) in various testicular cell types and is thought to exert its effects mainly via the action of RARs in Sertoli cells and early germ cells [2].

A previous study reported that the RAR-dependent effects of RA in Sertoli cells contribute to prepubertal testis development [22]. Moreover, as RA signaling in the testis is closely associated with the periodic cycle functions of Sertoli cells $[22,23]$, it is possible that vitamin A affects the regulation of BTB integrity by alteration of the seminiferous epithelial cycle. Indeed, in rats, vitamin A deprivation after weaning has been reported to induce disruption of the BTB [24, 25]. However, some reports have indicated that vitamin A deficiency has no effect on BTB preservation [26]. To clarify the importance of vitamin A in the maintenance of spermatogenesis during testes development, it is essential to assess spermatogenesis progression with changes in BTB integrity and the vitamin A pathway.

The present study assessed the impact of vitamin A deprivation on the murine seminiferous epithelium from the prepubertal period to adulthood, with a focus on spermatogenic progression and BTB assembly. The results showed that feeding the VAD diet to the parental generation (Fig. 1B) induced critical defects in spermatogenesis progression and altered the BTB integrity in adult testes compared with that in the prepubertal testes of the progeny. On the basis of the results of the present study, we propose that BTB integrity is regulated by vitamin A metabolism with control of meiosis and is required for persistent differentiation of spermatocytes rather than the initiation of meiosis.

\section{Materials and Methods}

\section{Mice}

Two-month-old male and female C57BL/6N mice were purchased from Japan SLC (Hamamatsu, Japan). VAD diet feeding and RA replacements were performed as described in a previous study [20]. Briefly, mice were fed a VAD diet (D13110GC; Research Diets, New Brunswick, NJ, USA). After 4-12 weeks of feeding this diet, the animals were used as breeding pairs. After weaning, male pups received the VAD diet until 90 days postpartum (dpp). Testes of mice fed the VAD diet and those fed a normal vitamin A-sufficient (VAS) diet (Labo MR Standard, NOSAN, Yokohama, Japan) were weighed, and epididymides and testes were harvested at 5-90 dpp. At $91 \mathrm{dpp}$, when the body weight was slightly decreased, all-trans-RA (Nacalai Tesque, Kyoto, Japan, $0.5 \mathrm{mg} /$ head) was intraperitoneally injected into mice fed the VAD diet, and the VAD diet was changed to the VAS diet. All-trans-RA was dissolved in a mixture of $25 \mu$ l ethanol and $75 \mu \mathrm{l}$ sesame oil (MP Biomedicals, Santa Ana, CA, USA). Testes were weighed and collected after 2-44 days of RA replacement (Fig. 1B). For analyses, mice were obtained by free breeding, maintained under specific pathogen-free conditions and sacrificed according to the Guide for the Care and Use of Laboratory Animals of Hokkaido University, Graduate School of Veterinary Medicine (approved by the Association for Assessment and Accreditation of Laboratory Animal Care International).

\section{Immunohistochemistry}

For immunohistochemistry (IHC), testes were removed and immersion-fixed in $4 \%(\mathrm{w} / \mathrm{v})$ paraformaldehyde $(\mathrm{PFA})$ in $0.1 \mathrm{M}$ phosphate buffer. Following fixation, testes were dehydrated in ethanol and embedded in paraffin. Sections were then deparaffinized and hydrated. Antigen retrieval was performed for $15 \mathrm{~min}$ at $105 \mathrm{C}$ with Target Retrieval Solution (DakoCytomation, Carpinteria, CA, USA) for OCLN and CLDN11 or with buffered citrate ( $\mathrm{pH}$ 6.0) for the following proteins: stimulated by retinoic acid gene 8 (STRA8), DMC1 dosage suppressor of mck1 homolog, meiosis-specific homologous recombination (DMC1), and synaptonemal complex protein 3 (SCP3, also known as SYCP3). Samples were treated with methanol containing $0.3 \% \mathrm{H}_{2} \mathrm{O}_{2}$ to eliminate endogenous peroxidase. After blocking with normal serum, sections were incubated with rabbit anti-OCLN $(1: 100$; 
Table 1. Primer pairs used in this study

\begin{tabular}{|c|c|c|c|}
\hline Gene name (Accession) & Symbol & Primer sequence $\left(5^{\prime}-3^{\prime}\right)$ & Product size (bp) \\
\hline $\begin{array}{l}\text { aldehyde dehydrogenase family } 1 \text {, subfamily A2 } \\
\text { (NM_009022) }\end{array}$ & Aldh1a2 & $\begin{array}{l}\text { F: TCTGTTGGACAAGCTTGCAG } \\
\text { R: CCAGCCTGCATAATACCTCAG }\end{array}$ & 148 \\
\hline $\begin{array}{l}\text { cytochrome P450, family } 26 \text {, subfamily a, polypeptide } 1 \\
\text { (NM_007811) }\end{array}$ & Cyp26al & $\begin{array}{l}\text { F: ATTGAGCACTCGTGGGAGAG } \\
\text { R: CTTCTCGAACTTTCTGGAGGAC }\end{array}$ & 163 \\
\hline $\begin{array}{l}\text { occludin } \\
\text { (NM_008756) }\end{array}$ & Ocln & $\begin{array}{l}\text { F: CCTACTCCTCCAATGGCAAA } \\
\text { R: CTCTTGCCCTTTCCTGCTTT }\end{array}$ & 208 \\
\hline $\begin{array}{l}\text { claudin } 3 \\
\left(\mathrm{NM} \_009902\right)\end{array}$ & Cldn3 & $\begin{array}{l}\text { F: GCACCCACCAAGATCCTCTA } \\
\text { R: TCGTCTGTCACCATCTGGAA }\end{array}$ & 206 \\
\hline $\begin{array}{l}\text { claudin } 11 \\
(\mathrm{NM} 008770)\end{array}$ & Cldn11 & $\begin{array}{l}\text { F: TGGTGGACATCCTCATCCTT } \\
\text { R: GCCAGCAGAATAAGGAGCAC }\end{array}$ & 190 \\
\hline $\begin{array}{l}\text { tight junction protein } 1(: \mathrm{ZO} 1) \\
(\mathrm{NM} \text { _009386) }\end{array}$ & Tjpl & $\begin{array}{l}\text { F: GCACCATGCCTAAAGCTGTC } \\
\text { R: ACTCAACACACCACCATTGC }\end{array}$ & 122 \\
\hline $\begin{array}{l}\text { actin, beta } \\
\text { (NM_007393) }\end{array}$ & $A c t b$ & $\begin{array}{l}\text { F: ACTGCTCTGGCTCCTAGCAC } \\
\text { R: CAGCTCAGTAACAGTCCGCC }\end{array}$ & 196 \\
\hline
\end{tabular}

F, forward; R, reverse; ZO1, zonula occludens 1 .

Life Technologies, Carlsbad, CA, USA), rabbit anti-CLDN11 (1:100; Life Technologies), rabbit anti-STRA8 (1:2000; Abcam, Cambridge, UK), goat anti-DMC1 (1:500; Santa Cruz Biotechnology, Santa Cruz, CA, USA), rabbit anti-SCP3 (1:800; Novus Biologicals, Littleton, CO, USA), and rabbit anti-single stranded DNA (ssDNA; 1:200; IBL, Fujioka, Japan) at 4 C overnight. Next, the sections were treated with biotin-conjugated goat anti-rabbit IgG antibodies (SABPO kit, Nichirei, Tokyo, Japan) or with biotin-conjugated donkey anti-goat IgG antibodies (1:100; Santa Cruz Biotechnology) for $30 \mathrm{~min}$ at room temperature, followed by treatment with streptavidin-biotin complex (SABPO kit) for $30 \mathrm{~min}$ at room temperature. The sections were incubated with 3,3'-diaminobenzidine tetrahydrochloride (DAB) solution containing $0.006 \% \mathrm{H}_{2} \mathrm{O}_{2}$ until the stain developed and were then counterstained with hematoxylin. For histometric analyses, BZ-9000 (Keyence, Osaka, Japan) was used for acquisition of digital images of each IHC section, and the immunopositive cells were counted with the BZ-II Analyzer software (Keyence).

\section{Histopathology}

Paraffin sections were prepared from testes and epididymides fixed in 4\% PFA fixed as described above for periodic acid-Schiffhematoxylin (PAS-H) or von Kossa staining. For von Kossa staining, sections were incubated with $5 \%$ silver nitrate solution under sunlight for $60 \mathrm{~min}$. The slides were then washed in water, differentiated with $5 \%$ sodium thiosulfate pentahydrate for $3 \mathrm{~min}$, rinsed, and counterstained for $5 \mathrm{~min}$ with nuclear fast red.

\section{Reverse transcription-PCR and quantitative real-time PCR}

Total RNA was extracted from whole testes harvested from mice fed the VAD diet at 5-90 dpp and at 16-44 days after RA replacement using TRIzol reagent (Life Technologies). Purified total RNA was treated with Turbo DNase (Life Technologies) for DNA digestion, and complementary DNAs (cDNAs) were synthesized via reverse transcription (RT) reactions using ReverTra Ace (Toyobo, Osaka, Japan) and oligo-dT primers (Life Technologies). Quantitative real-time PCR (qPCR) analysis subsequent to RT was performed using the prepared cDNA, gene-specific primers (Table 1), Brilliant III Ultra Fast SYBR Green QPCR Master Mix (Agilent Technologies, Santa Clara, CA, USA) and a real-time thermal cycler (MX 3000P; Agilent Technologies). Levels of mRNA expression of the target genes were normalized to those of actin, beta.

\section{Statistical analyses}

Results are expressed as the means \pm standard errors (SEs), and statistical analyses were performed using PASW Statistics for Windows, Version 18.0 (IBM SPSS, Chicago, IL, USA). The Mann-Whitney $U$ test was used for comparison between 2 groups. One-way analysis of variance (ANOVA) followed by Dunnett's test was used to compare changes between treatment groups and corresponding untreated groups. P-values less than 0.05 were considered statistically significant.

\section{Results}

Changes in the weight of the testes in mice fed the VAD diet

The changes in the weight of testes collected from mice fed the VAD diet and control mice fed the VAS diet are shown in Fig. 2A. From 5-50 dpp, the weight of testes of the VAD diet group increased similarly to that of the control group, indicating that prepubertal testis development in mice fed the VAD diet proceeded normally until approximately $50 \mathrm{dpp}$. However, the weight of the testes collected from mice fed the VAD diet gradually decreased from 60 dpp onwards. At 70-90 dpp, just before RA administration, the weight of the testes collected from mice fed the VAD diet decreased significantly compared with that of control mice.

\section{Effects of VAD diet feeding on RA signaling in the mouse} testes

To assess the functional abnormalities of RA signaling in mice fed the VAD diet, mRNA levels of RA signaling molecules were analyzed in the testes of prepubertal mice (0-20 dpp) and sexually mature mice (90 dpp). Aldehyde dehydrogenase family 1, subfamily A2 

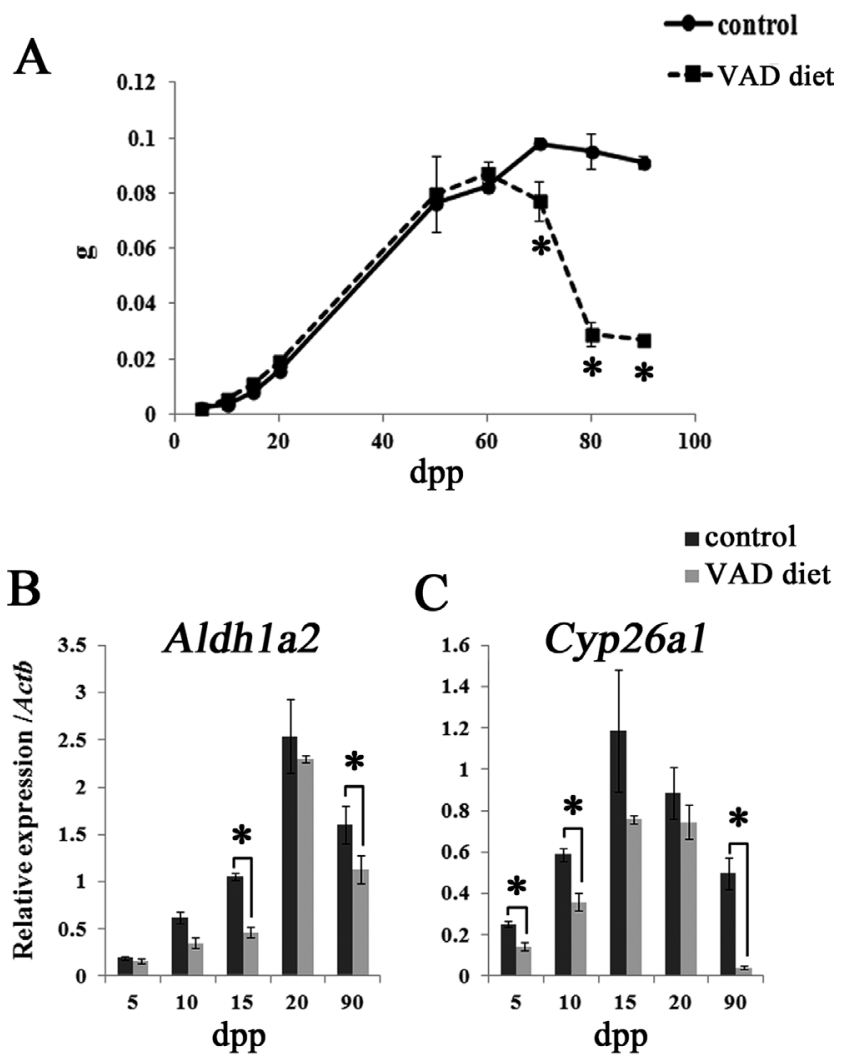

Fig. 2. Testes weight and the expression of RA signaling molecule transcripts in the testes of mice fed the VAD diet. A: Changes in the testes weight of mice fed the VAD diet and control mice fed the VAS diet. Values shown are means \pm SEs. * Significant difference between control mice and mice fed the VAD diet $(\mathrm{P}<0.05$, Mann-Whitney $U$ test $)$. B-C: qPCR analyses of Aldh1a2 (B) and Cyp26al mRNAs (C) during the prepubertal period (5-20 dpp) and adulthood (90 dpp) in mice fed the VAD diet and control mice fed the VAS diet. Values shown are means \pm SEs. $* \mathrm{P}<0.05$, Mann-Whitney $U$ test.

(Aldh1a2) and cytochrome P450 family 26, subfamily a, polypeptide 1 (Cyp26a1) were examined as RA signaling molecules (Fig. 2B and C). Aldh1 2 mRNA levels were lower in mice fed the VAD diet than in control mice, and statistically significant differences were detected at 15 and $90 \mathrm{dpp}$ (Fig. 2B). Furthermore, significant decreases in Cyp26a1 mRNA expression in the testes of mice fed the VAD diet were detected at 5, 10, and $90 \mathrm{dpp}$, with the most dramatic decrease observed at $90 \mathrm{dpp}$ (Fig. 2C).

\section{Histological abnormalities of the testes and epididymides in mice fed the VAD diet}

Although the seminiferous epithelium showed no significant histological differences between control mice and mice fed the VAD diet during the prepubertal period (at $10 \mathrm{dpp}$ as shown in Fig. 3A and B), the seminiferous epithelium of mice fed the VAD diet showed severe histological abnormalities characterized by epithelial vacuolization observed from $70 \mathrm{dpp}$ (Fig. 3D, arrows). This vacuolization was barely detectable in testes from control mice throughout the observation period (Fig. 3C). By 80 dpp, progressive atrophy with marked depletion of germ cells was observed in all seminiferous tubules of mice fed the VAD diet (Fig. 3E). Moreover, from 80 dpp onwards, von Kossa staining revealed that dystrophic calcification occurred in the severely degenerated tubules of mice fed the VAD diet (Fig. 3F). Intratubular calcification was observed in $6.78 \% \pm 3.62 \%$ of seminiferous tubules of mice fed the VAD diet $(\mathrm{n}=3)$ at $80 \mathrm{dpp}$, and this increased to $19.34 \% \pm 3.60 \%(\mathrm{n}=4)$ at 90 dpp. In the epididymides of mice fed the VAD diet, no significant histological changes were observed, and the epididymides contained spermatozoa, as expected, until $70 \mathrm{dpp}$ (Fig. 3G). However, severe squamous metaplasia was frequently observed in the corpus epididymis from 80 dpp onwards (Fig. 3H).

\section{Induction of germ cell apoptosis in the testes of mice fed the $V A D$ diet}

To elucidate whether germ cell apoptosis was induced concurrently with testicular degeneration in mice fed the VAD diet, IHC analysis for ssDNA, an apoptotic cell marker, was performed (Fig. 4). In prepubertal testes (5-20 dpp), a number of ssDNA-positive cells were observed in both control mice and mice fed the VAD diet (Fig. 4A). From 50-90 dpp, testes from control mice continually showed few ssDNA-positive cells in the seminiferous tubules (Fig. 4A and B). In contrast, testes from mice fed the VAD diet contained numerous ssDNA-positive cells from 50-70 dpp, especially in the luminal parts of seminiferous tubules. These apoptotic cells seemed to be mainly pachytene spermatocytes and round spermatids, reflecting the apoptotic induction in germ cells (Fig. 4C). In a histometric analysis, mice fed the VAD diet showed a higher number of ssDNA-positive cells as compared with control mice from 50-70 dpp, and a statistically significant difference was observed at $60 \mathrm{dpp}$ (Fig. 4A).

\section{Effects of the VAD diet on the progression of meiosis}

To elucidate the effects of vitamin A insufficiency on the seminiferous epithelium, the progression of meiosis was examined from the prepubertal period to adulthood (Fig. 5). For IHC analysis, STRA8, DMC1 and SYCP3 were used as markers of preleptotene and early leptotene spermatocytes, leptotene and zygotene spermatocytes, and leptotene to metaphase I spermatocytes, respectively (Fig. 1A) [27-32]. Figures 5A-C show the time course of immunopositive cell numbers in mouse testes. STRA8-positive cells were detected from $10 \mathrm{dpp}$ in the testes of mice fed the VAD diet and control mice (Fig. 5A). However, the number of STRA8-positive cells in mice fed the VAD diet tended to be lower than that in control mice throughout the observation period (Figs. 5A and D-G), and significant differences were observed at 15, 70, 80 and $90 \mathrm{dpp}$ (Fig. 5A). However, DMC1- and SYCP3-positive cells were detected at similar levels from 15-50 dpp in the testes of mice fed the VAD diet and control mice (Figs. 5B and C, Fig. 5H vs. 5I and Fig.5L vs. 5M). The numbers of DMC1 - or SYCP3-positive cells were significantly decreased in mice fed the VAD diet from 70 and 60 dpp onwards, respectively (Figs. 5B and 5C, Fig. 5J vs. $5 \mathrm{~K}$ and Fig. $5 \mathrm{~N}$ vs. 5O). After $80 \mathrm{dpp}$, it was difficult to detect immunopositive spermatocytes for all examined markers in the testes of mice fed the VAD diet, reflecting spermatogenic arrest (Figs. 5A-C). 


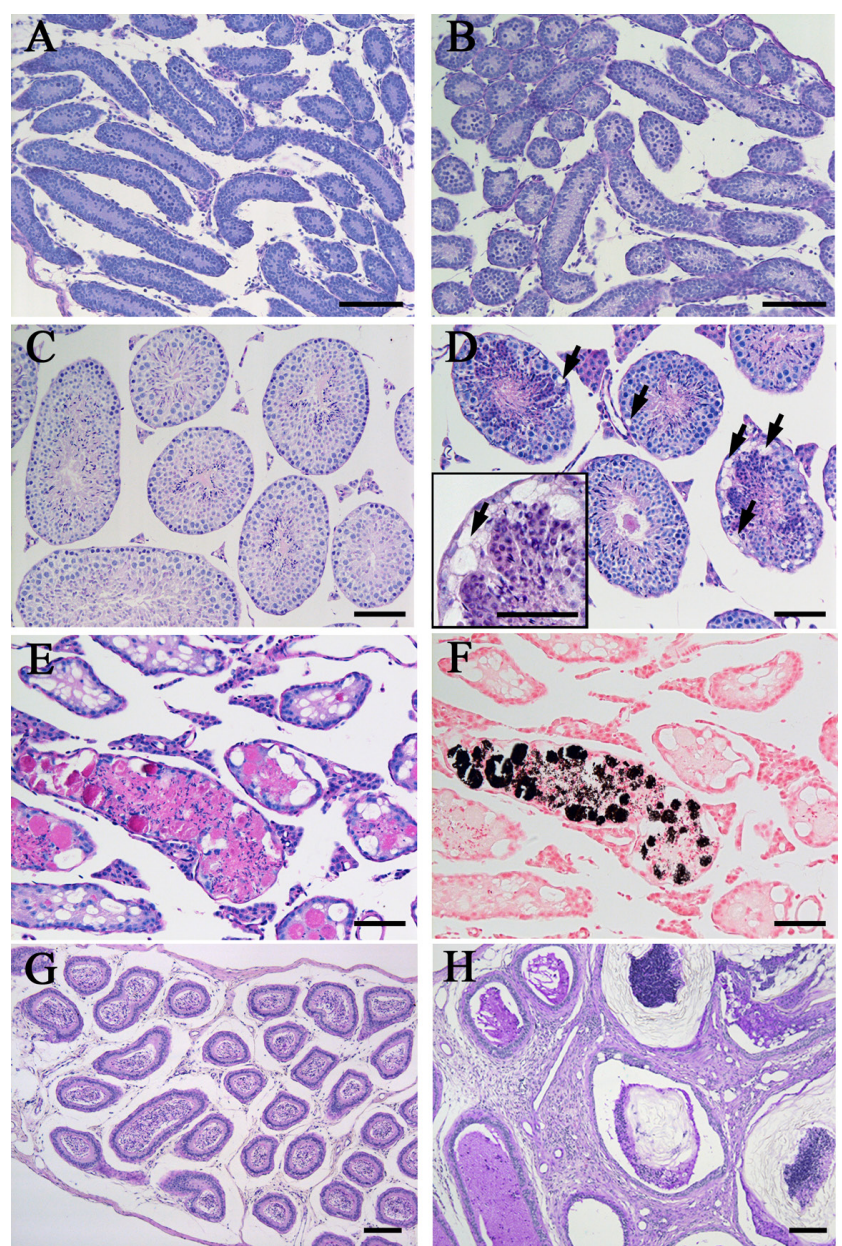

Fig. 3. Histopathological abnormalities in the seminiferous epithelium and epididymides of mice fed the VAD diet. (A-F) Histological features of seminiferous tubules in mice fed the control VAS diet and those fed the VAD diet. Panels A-E and F show PAS-H and von Kossa staining, respectively. At $10 \mathrm{dpp}$, no differences were observed between the testes of control mice (A) and mice fed the VAD diet (B). In testes from control mice at $90 \mathrm{dpp}$, no histopathological abnormalities were detected $(C)$. Some tubules in the testes of mice fed the VAD diet showed epithelial vacuolization at $70 \mathrm{dpp}(\mathrm{D}$, arrows). The insert box in panel $\mathrm{D}$ highlights the epithelial vacuolization at the basal regions of a tubule (arrow). Progressive atrophy of all tubules with marked depletion of germ cells was observed at $80 \mathrm{dpp}$ in testes from mice fed the VAD diet (E). In serial sections of panel E, von Kossa-positive calcification was detected in severely degenerated tubules (F). (G-H) PAS-H-stained epididymides of mice fed the VAD diet. No significant abnormalities were detected at $70 \mathrm{dpp}$, and spermatozoa were observed in the lumen of ducts $(G)$. Severe squamous metaplasia in the corpus epididymis was observed at 80 dpp (H). Bars $=100 \mu \mathrm{m}$.

\section{Assembly and disruption of the BTB in the testes of mice fed} the VAD diet

To assess the effects of the VAD diet on BTB assembly, the mRNA expression of BTB component TJ genes, including Ocln, Cldn3, Cldn11, and Tjp1 (encoding ZO1), was evaluated in the testes in the prepubertal period (5-20 dpp) and at a sexually mature age (90
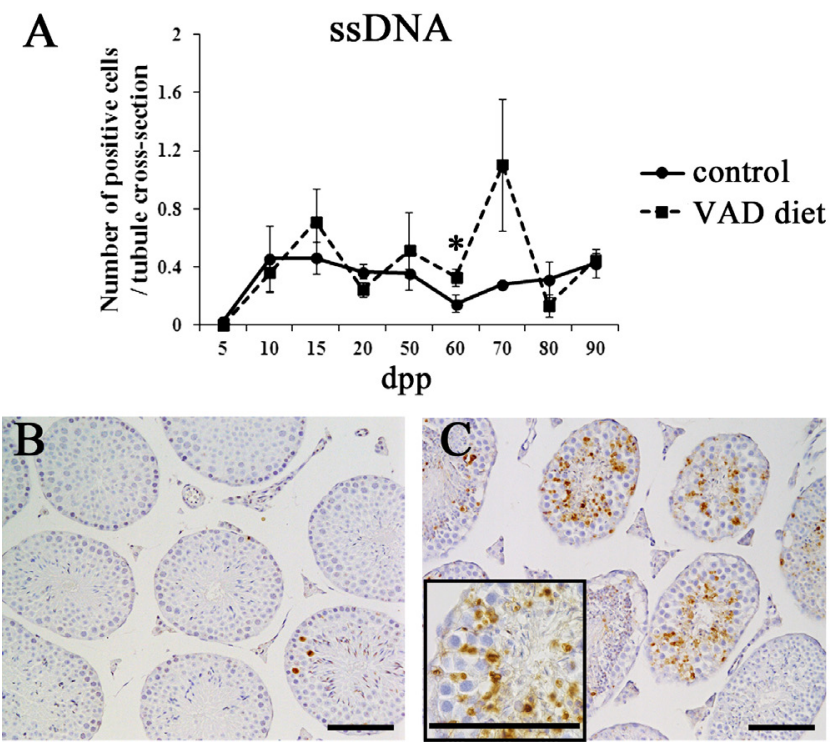

Fig. 4. Germ cell apoptosis in the testes of mice fed the VAD diet. (A) Total numbers of ssDNA-positive cells in the testes of control mice fed the VAS diet and mice fed the VAD diet. ssDNA-positive cells were counted in 200-500 tubules per testis of control mice and mice fed the VAD diet from 5-90 dpp using IHC sections. Values shown are means \pm SEs. *Significant difference between control mice and mice fed the VAD diet $(\mathrm{P}<0.05$, Mann-Whitney $U$ test). (B-C) Appearance of ssDNA-positive cells in the seminiferous epithelium. ssDNA-positive cells were only infrequently detected in testes from control mice at $70 \mathrm{dpp}$ (B). Testes from mice fed the VAD diet contained numerous ssDNA-positive cells at $70 \mathrm{dpp}$ (C). The insert box in panel $\mathrm{C}$ highlights the ssDNA-positive cells in the luminal area of the seminiferous tubules. Bars $=100 \mu \mathrm{m}$.

dpp; Fig. 6). Consistent with previous reports [8, 10, 12], mRNA expression of the above genes in control mice tended to increase during the prepubertal period (5-20 dpp) and then decreased by sexual maturation (90 dpp; Figs. 6A-D). In mice fed the VAD diet, the mRNA expression of these genes tended to be lower than that in control mice from 5-20 dpp (Figs. 6A-D). Significant differences in the expression of Ocln mRNA at $5 \mathrm{dpp}, C l d n 11$ mRNA at $15 \mathrm{dpp}$ and Tjp 1 mRNA at 5 and $15 \mathrm{dpp}$ were observed between mice fed the VAD diet and control mice (Figs. 6A, C and D). At 90 dpp, the mRNA expression levels of Ocln and Cldn 11 were significantly higher in mice fed the VAD diet than in control mice (Figs. 6A and C). No significant changes were observed in Cldn 3 expression between mice fed the VAD diet and control mice throughout the observation period (Fig. 6B).

To evaluate BTB integrity, OCLN and CLDN11 localization was compared between testes from control mice and mice fed the VAD diet from the prepubertal period to adulthood (Figs. 6E-N). Consistent with a previous study [11], OCLN was weakly and diffusely distributed from the apical to basal regions of Sertoli cells in control mice at $10 \mathrm{dpp}$ (Fig. 6E) and began to accumulate in the basal part of the seminiferous tubules from $15 \mathrm{dpp}$ (Fig. $6 \mathrm{~F})$. In mice fed the VAD diet, OCLN showed a weak and diffuse pattern in seminiferous tubules, even at 15 dpp (Fig. 6J), whereas OCLN seemed to localize to the basal portion of the seminiferous 



Fig. 5. Meiotic progression in the testes of mice fed the VAD diet. (A-C) The numbers of STRA8-positive cells (A), DMC1positive cells (B) and SYCP3-positive cells (C) in the testes of control mice fed the VAS diet and mice fed the VAD diet. Positive cells were counted in 200-500 tubules per testis. Values shown are means \pm SEs. * Significant difference between control mice and mice fed the VAD diet $(\mathrm{P}<0.05$, Mann-Whitney $U$ test). (D-G) The appearance of STRA8-positive cells in the seminiferous tubules. Cells positive for STRA8, a marker for preleptotene and early leptotene spermatocytes, were observed at 15 (D) and $70 \mathrm{dpp}$ (F) in control testes. In testes from mice fed the VAD diet, although STRA8-positive cells were observed at $15 \mathrm{dpp}(\mathrm{E})$, they were barely detectable at $70 \mathrm{dpp}(\mathrm{G})$. (H-K) The appearance of DMC1-positive cells in the seminiferous tubules. Cells positive for DMC1, a marker for leptotene and zygotene spermatocytes, were observed at similar levels at $15 \mathrm{dpp}$ in control testes (H) and testes from mice fed the VAD diet (I). In contrast, at $70 \mathrm{dpp}$, DMC1-positive cells were decreased in mice fed the VAD diet (K) compared with control mice (J). (L-O) The appearance of SYCP3-positive cells in the seminiferous tubules. Cells positive for SYCP3, a marker for leptotene to metaphase I spermatocytes, were detected in almost all tubules in both the control mice (L) and mice fed the VAD diet (M) at $15 \mathrm{dpp}$ and in control mice only at $70 \mathrm{dpp}(\mathrm{N})$. SYCP3-positive cells were not detectable in some tubules of testes from mice fed the VAD diet at $70 \mathrm{dpp}(\mathrm{O})$. Bars $=50 \mu \mathrm{m}$.

tubules by $20 \mathrm{dpp}$ (data not shown). During 10-20 dpp, a similar localization pattern to OCLN was observed in CLDN11 (data not shown). In control mice at $60 \mathrm{dpp}$, OCLN localized to the basal side of leptotene or zygotene spermatocytes in stage X tubules (Fig. 6G, arrows), indicating normal migration of spermatocytes across the BTB. However, in stage X tubules of mice fed the VAD diet, OCLN localized to the luminal side as well as the basal side of leptotene or zygotene spermatocytes at $60 \mathrm{dpp}$ (Fig. 6K, arrowheads). In mice fed the VAD diet, OCLN positivity disappeared with severe epithelial vacuolization at the basal regions of tubules at $70 \mathrm{dpp}$ (Fig. 6L), indicating BTB disruption. Furthermore, in testes from mice fed the VAD diet at $90 \mathrm{dpp}$, Sertoli cell detachment from the basement membrane was observed in $25.58 \% \pm 3.06 \%$ tubules $(n=3)$, and OCLN- and CLDN11-positive staining was diffusely observed in abnormal tubules at $90 \mathrm{dpp}$ (Fig. $6 \mathrm{H}$ vs. 6M, Fig. 6I vs. 6N). 



Fig. 6. BTB integrity in the testes of mice fed the VAD diet. (A-D) mRNA levels of $O c \ln (\mathrm{A}), C l d n 3$ (B), $C l d n 11$ (C), and Tjp1 (D) in the testes of control mice fed the VAS diet and mice fed the VAD diet during the prepubertal period (5-20 dpp) and adulthood ( 90 dpp). Values shown are means \pm SEs. *Significant difference between control mice and mice fed the VAD diet $(\mathrm{P}<0.05$, Mann-Whitney $U$ test). (E-N) BTB localization in control mice and mice fed the VAD diet. Positive reactions were detected by IHC for OCLN (E-H and J-M) and CLDN11 (I and N). In testes from control mice, specific OCLN localization was barely detectable at $10 \mathrm{dpp}$ (E), while OCLN was accumulated in the basal part of the seminiferous tubules at $15 \mathrm{dpp}(\mathrm{F})$. Few immunoreactions for OCLN were observed at $15 \mathrm{dpp}$ in testes from mice fed the VAD diet (J). In stage $\mathrm{X}$ tubules of control mice at $60 \mathrm{dpp}, \mathrm{OCLN}$ localized only to the basal side of leptotene or zygotene spermatocytes (G, arrows). In stage $\mathrm{X}$ tubules of mice fed the VAD diet at $60 \mathrm{dpp}$, OCLN localized to the luminal side of leptotene or zygotene spermatocytes (K, arrowheads). At $70 \mathrm{dpp}$ in testes from mice fed the VAD diet, immunopositive OCLN signals decreased, and epithelial vacuolization was observed (L). At $90 \mathrm{dpp}$ in testes from control mice, Sertoli cell nuclei were aligned along the basement membrane of tubules with OCLN (H) and CLDN11 (I) localization. In contrast, testes from mice fed the VAD diet showed diffuse distribution of OCLN (M) and CLDN11 (N) with detached Sertoli cells at $90 \mathrm{dpp}(\mathrm{M}$ and $\mathrm{N}$, arrows). Bars $=50 \mu \mathrm{m}$.

Re-initiation of spermatogenesis and reassembly of the BTB after $R A$ replenishment in mice fed the VAD diet

As shown in Fig. 7, re-initiation of spermatogenesis was evaluated with the assembly of the BTB after RA administration and dietary vitamin A replenishment in adult mice fed the VAD diet (see also Fig. 1B). From 91 dpp, day 0 after RA replenishment, weight of the testes in mice fed the VAD diet gradually increased throughout the observation period (Fig. 7A). After RA replenishment, the number of seminiferous tubules containing the germ cells increased with the increase of the diameter (Fig. 7B vs. 7C). Furthermore, at day 35 after RA replenishment, spermatogenesis progressed to step 16 spermatids, germ cells just prior to spermiation (Fig. 7C, arrows). These observations reflected the re-initiation of spermatogenesis after RA replenishment, which is consistent with a previous report [19].
Despite the re-initiation of spermatogenesis, intratubular calcification was detected, even after RA replenishment, throughout the observation period (Fig. 7B vs. 7C), and the incidence rate of von Kossa-positive seminiferous tubules did not change significantly before or after RA replenishment $(14.33 \% \pm 1.59 \%$ at day $34-36, n=12)$. The levels of mRNA expression of $O c l n$ and $C l d n 11$ were significantly decreased in a time-dependent manner after RA replenishment (Figs. 7D and F), but no significant changes were observed in the levels of $\mathrm{Cldn} 3$ and Tjp1 mRNAs (Figs. 7E and G). In IHC analysis, although OCLN and CLDN11 positivity was unclear and scattered in the seminiferous epithelium until day 9 after RA replenishment (Figs. $7 \mathrm{H}, \mathrm{I}, \mathrm{L}$ and M), OCLN and CLDN11 localized to the proper basal portions of the seminiferous tubules with the emergence of pachytene and preleptotene/leptotene spermatocytes (Figs. 7J and N, arrows 
A

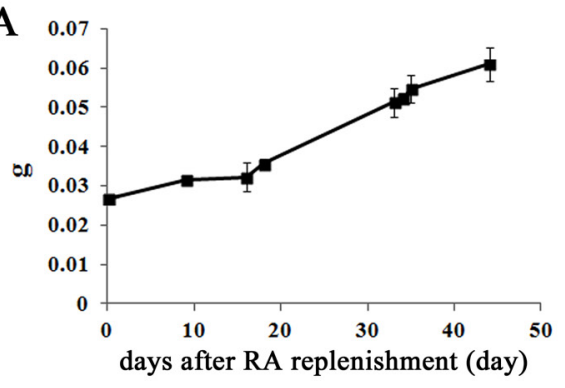

D

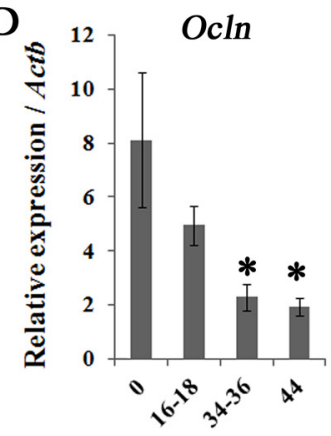

day

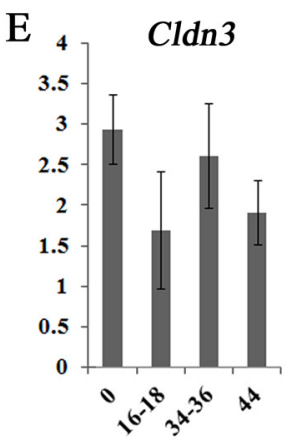

day
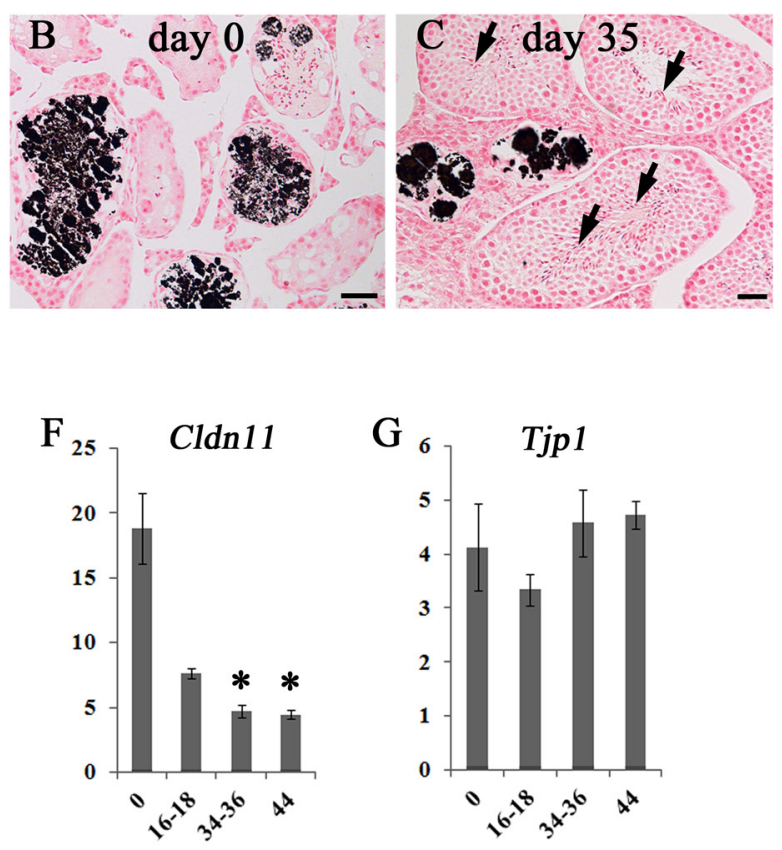

day

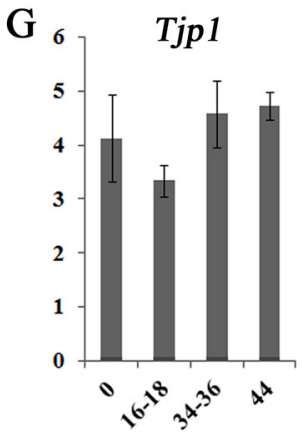

day
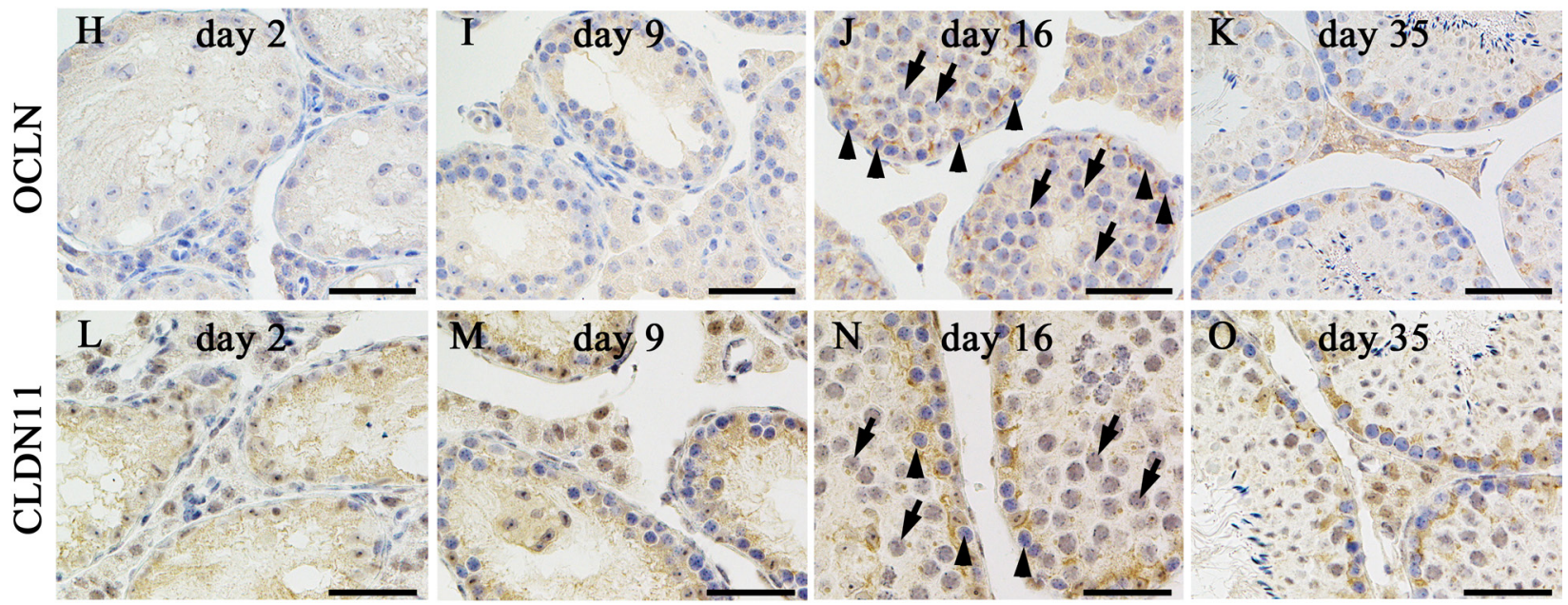

Fig. 7. Effects of RA replenishment on the testes of mice fed the VAD diet. (A) Changes in testes weight after RA replenishment in mice fed the VAD diet. Values shown are means \pm SEs. (B-C) Testes of mice fed the VAD diet after RA replenishment. Intratubular calcifications were observed by von Kossa staining on day 0 (B) and day 35 (C). At day 35, the majority of tubules contained step 16 spermatids (C, arrows). Bars $=50 \mu \mathrm{m}$. (D-G) mRNA levels of $O c \ln (\mathrm{D}), \operatorname{Cldn} 3$ (E), Cldn11 (F), and Tjpl (G) in the testes of mice fed the VAD diet after RA replenishment. Values shown are means \pm SEs. * Significant difference between control mice and mice fed the VAD diet $(\mathrm{P}<0.05$, one-way ANOVA followed by Dunnett's test). (H-O) BTB organization in the testes of mice fed the VAD diet after RA replenishment. An OCLN-positive signal was barely detectable at days 2 (H) and 9 (I) after RA replenishment but was clearly localized to the basal part of seminiferous tubules at day $16(\mathrm{~J})$, with the presence of pachytene spermatocytes $(\mathrm{J}$, arrows) and preleptotene/leptotene spermatocytes (J, arrowheads). Similarly, s CLDN11-positive signal was diffused at day 2 (L) and day 9 (M) after RA replenishment, but was clearly localized to the basal part of seminiferous tubules at day $16(\mathrm{~N})$, with the presence of pachytene spermatocytes (N, arrows) and preleptotene/ leptotene spermatocytes (N, arrowheads). Normal localization of OCLN and CLDN11 in the basal part of the tubules was also observed at day $35(\mathrm{~K}$ and $\mathrm{O})$. Bars $=50 \mu \mathrm{m}$.

and arrowheads, respectively) from approximately day 16 after RA replenishment. Subsequently, stage-specific changes in OCLN and CLDN11 localization were typically observed in RA-replenished testes (Figs. 7K and 7O).

\section{Discussion}

Efficiency of the VAD diet feeding strategy

In the male mouse, meiosis is initiated after birth and continues throughout the reproductive period. Increasing evidence has indicated 
that vitamin A is required for the maintenance of spermatogenesis [2]. During prepubertal testis development, neonates receive vitamin A from the maternal milk. Vitamin A is stored primarily in the liver and, to a lesser extent, in other tissues. To induce vitamin A insufficiency in the prepubertal period itself, we fed parental mice the VAD diet from at least 4 weeks prior to mating (Fig. 1B) as previously described [20]. Mice fed the VAD diet showed reduced expression of RA signaling molecule transcripts (i.e., Aldh1a2 and Cyp26a1) compared with those in control mice fed the VAS diet during the prepubertal period (5-20 dpp). Furthermore, dramatic decreases in the expression levels of these genes were observed at adulthood (90 dpp) in mice fed the VAD diet. RA synthesis from vitamin A is typically controlled by tissue-specific activity of retinaldehyde dehydrogenase (RALDH). In the testes, Aldh1a2 (encoding RALDH2) is expressed in Sertoli and germ cells [23]. RA is then oxidized into its inactive forms by CYP26, and Cyp26al is expressed by Sertoli cells and transcribed by the activation of RA signaling [23]. Taken together, these findings suggest that functional abnormality of RA signaling is induced beginning in the prepubertal period and that the effects of VAD were critical at $90 \mathrm{dpp}$ in the present VAD diet feeding strategy.

\section{Seminiferous epithelial disruption with germ cell apoptosis in testes of mice fed the VAD diet}

In mice fed the VAD diet, histological changes characterized by vacuolization of the seminiferous epithelium were observed from 70 dpp onwards. This observation corresponded to a significant reduction in weight of the testes in mice fed the VAD diet. Vacuolization of the seminiferous epithelium is a well-known response of Sertoli cells to various kinds of damages, and often occurs before extensive germ cell degeneration [33-37]. RA has been suggested to mainly exert its effects via the action of RARs in Sertoli cells and germ cells [2]; therefore, we speculate that the VAD condition would directly cause vacuolization in the seminiferous epithelium. However, no vacuolization was observed during the prepubertal period in the testes of mice fed the VAD diet. Furthermore, massive apoptotic induction of germ cells was observed from $60 \mathrm{dpp}$ onwards, and spermatogenesis was almost completely halted at the spermatogonial stages by $80 \mathrm{dpp}$. This germ cell death would likely contribute to the dramatic decrease in weight of the testes in adult mice fed the VAD diet. Although the efficiency of VAD may cause differences between the prepubertal period and adulthood, these results reflected differences in the effects of RA during testicular development.

Unexpectedly, we noted calcification in severely degenerated seminiferous tubules of mice fed the VAD diet from 80 dpp onwards. In clinical reports in humans, testicular calcification has been detected in association with several pathological conditions, including infertility, cryptorchidism, testicular neoplasms, Klinefelter's syndrome, varicocele, and torsion of the testis [38-41], and in normal testes [42]. However, the etiology of testicular calcification remains to be determined. Recently, intratesticular calcification has been reported to occur in an age-dependent manner in mutant mice showing Sertoli cell dysfunction with progressive germ cell degeneration [35, 43, 44]. In the present study, adult mice fed the VAD diet showed Sertoli cell detachment from the basement membrane of seminiferous tubules. Therefore, these data suggested that the VAD condition induced abnormal polarity and dysfunction of Sertoli cells. Intratubular calcification was detectable even after RA replenishment, indicating that the resumption of spermatogenesis could no longer occur in specific parts of the tubules. Indeed, in the rat testes, prolonged deprivation of vitamin A beyond 10-11 weeks has been shown to result in permanent failure of spermatogenesis, despite RA replenishment [21]. Taken together, these data suggest that the onset of calcification in the seminiferous epithelium could reflect the irreversible damage caused by long-term vitamin A deprivation.

\section{Delayed spermatogenesis and BTB assembly in testes from prepubertal mice fed the VAD diet}

At $10 \mathrm{dpp}$, STRA8-positive (preleptotene or early-leptotene) spermatocytes derived from the first wave were detected at similar levels in testes from control mice and mice fed the VAD diet. However, in mice fed the VAD diet, the number of STRA8-positive cells tended to be lower than that in control mice from 15 dpp onwards, and a significant difference was observed at $15 \mathrm{dpp}$. Stra 8 is an RA-stimulated gene controlling the transition from the preleptotene to leptotene spermatocyte [45]. Therefore, the functional abnormality of RA signaling in mice fed the VAD diet might affect the STRA8 expression in some of the preleptotene or leptotene spermatocytes. However, we confirmed that mice fed the VAD diet showed STRA8 expression in all of the preleptotene and early leptotene spermatocytes distinguishable by the typical chromatin pattern of these cells using immunofluorescence analysis (data not shown). As shown in Fig. 1A, normal murine seminiferous tubules at $15 \mathrm{dpp}$ contained pachytene spermatocytes accompanying preleptotene/early-leptotene spermatocytes derived from the first and second waves, respectively $[46,47]$. Therefore, the reduced number of STRA8-positive cells from $15 \mathrm{dpp}$ may reflect suppressed meiosis initiation or onset of spermatogonial differentiation from the second wave onwards. Despite the difference in the number of STRA8-positive cells, mice fed the VAD diet showed no differences in the numbers of DMC1-positive cells (leptotene and zygotene spermatocytes) or SYCP3-positive cells (leptotene to metaphase I spermatocytes) up to $50 \mathrm{dpp}$. Furthermore, up to $70 \mathrm{dpp}$, no abnormal changes were observed in the epididymides of mice fed the VAD diet, and these epididymides contained spermatozoa, as under normal conditions. Consistent with our results, RAR $\alpha$-deficient mice, which exhibit progressive breakdown of the spermatogenic process, showed a normal number of pachytene spermatocytes at 4 weeks of age regardless of delay in the onset of the second wave [47]. Therefore, the imbalance between the numbers of STRA8-, DMC1-, and SYCP3-positive cells in mice fed the VAD diet up to $50 \mathrm{dpp}$ might reflect the abnormal progression of spermatogenesis. Importantly, mRNA expression levels of BTB components, including Ocln, Cldn11 and Tjp1, were significantly reduced in mice fed the VAD diet compared with those in control mice at 5-15 dpp. Furthermore, although the BTB was formed by $15 \mathrm{dpp}$ in normal mice, as previously described [7, 12], the initial assembly of the BTB, estimated by OCLN and CLDN11 positivity in the basal portion of seminiferous tubules, was barely detectable at $15 \mathrm{dpp}$ and delayed until $20 \mathrm{dpp}$ in testes from mice fed the VAD diet. Because OCLN and CLDN11 are principal components of the BTB in mouse testes [4], loss of proper localization of these TJ proteins indicates defective BTB fence function. These findings 
suggest that the VAD condition in prepubertal mice delayed both the appearance of STRA8-positive spermatocytes (preleptotene or early-leptotene) from the second wave and the initial formation of the BTB, although meiosis of the first wave and subsequent spermatogenesis from zygotene spermatocytes proceeded until $50 \mathrm{dpp}$.

After re-initiation of spermatogenesis by RA replenishment in spermatogenic-arrested testes of mice fed the VAD diet, it took 35 days for spermatogonia to differentiate into step 16 spermatids. Furthermore, at day 16 after RA replenishment, almost all tubules, except for calcified tubules, contained germ cells that had progressed to pachytene and preleptotene/leptotene spermatocytes. Because the differentiation from A1 spermatogonia to spermatozoa requires approximately 35 days during normal spermatogenesis [2], spermatogenesis in mice fed the VAD diet proceeded normally soon after RA replenishment. However, although Ocln expression in the testis was reported to be activated by RA signaling [10], RA did not immediately rescue OCLN and CLDN11 localization to the basal portions of seminiferous tubules in mice fed the VAD diet until day 16 after RA replenishment. Interestingly, at day 16, almost all tubules contained pachytene and preleptotene/leptotene spermatocytes. As mentioned above, at $15 \mathrm{dpp}$, the majority of seminiferous tubules contained pachytene spermatocytes from the first wave together with preleptotene/leptotene spermatocytes from the second wave $[46,47]$. In the seminiferous epithelium, Sertoli and germ cells coordinately establish an intimate and elaborate cellular network for cell-cell communications and regulate each other's functions via bidirectional trafficking [3]. Taken together, these findings and our results from the VAD diet study strongly suggest that the presence of spermatocyte sets derived from both the first and second wave, especially pachytene spermatocytes from the first wave and preleptotene/leptotene spermatocytes from the second wave, are required for the initial establishment of the BTB. Furthermore, RA signals may play a crucial role in regulating these processes during the prepubertal period.

\section{Failure of spermatogenesis and disrupted BTB integrity in} mice fed the VAD diet

In mice fed the VAD diet, a dramatic induction of germ cell apoptosis occurred from $60 \mathrm{dpp}$ onwards, and spermatogenesis was almost completely halted at the spermatogonial stages by $80 \mathrm{dpp}$. We observed aberrant OCLN localization to the luminal side of leptotene or zygotene spermatocytes in stage $\mathrm{X}$ tubules of mice fed the VAD diet at $60 \mathrm{dpp}$, indicating disruption of BTB integrity. Recently, stage-specific expression of OCLN was found to be regulated by RA signaling, and knockdown of Ocln was shown to induce apoptosis in germ cells in stage IX-XII tubules [10]. On the basis of these findings, the VAD diet was expected to induce apoptosis in germ cells in adulthood by altering the stage-specific localization of TJ proteins. Interestingly, zygotene or pachytene spermatocytes are thought to mainly be affected by incomplete BTB assembly [6]. Indeed, in testes from mice fed the VAD diet, although the number of SYCP3-positive cells (leptotene to metaphase I spermatocytes) decreased from 60 dpp onwards, STRA8- (preleptotene and earlyleptotene spermatocytes) or DMC1-positive cells (leptotene and zygotene spermatocytes) decreased from $70 \mathrm{dpp}$ onwards. These results indicated that germ cell apoptosis was primarily induced from the pachytene spermatocyte stage onwards, consistent with the observations in Fig. 4 and subsequently during the former stages of spermatocytes due to BTB disruption resulting from vitamin A deprivation.

In mice fed the VAD diet, mRNA expression levels of Ocln and Cldn11 significantly increased at $90 \mathrm{dpp}$ and gradually decreased after re-initiation of spermatogenesis by RA replenishment. These changes may be attributable simply to alterations in testicular cellularity (i.e., the ratio of somatic cells to germ cells). However, because postmeiotic germ cells, especially spermatids, have been reported to inhibit CLDN11 expression in rat testes [48], higher expression of TJ genes at $90 \mathrm{dpp}$ in mice fed the VAD diet may also reflect the loss of postmeiotic germ cells.

Despite the delayed and incomplete BTB formation, spermatogenesis in mice fed the VAD diet successfully proceeded up to 50 dpp but was arrested at a later period. The delayed and incomplete BTB formation and age-dependent testicular atrophy observed in the present study were consistent with results reported in mice with selective ablation of the androgen receptor in Sertoli cells [7, 44, 49]. These findings suggested that functional correlations between RA and sex hormones and an altered balance of these functions are critical for inducing seminiferous epithelial damage in adulthood. It is noteworthy that, similar to our results, Ocln-null mice showed typical testicular development with a normal set of germ cells at 6 weeks of age and later developed testicular atrophy and became sterile [15]. Furthermore, spermatogenesis of Cldn11-null mice progressed to round spermatids at $28 \mathrm{dpp}$ [50], after which the mice became sterile due to BTB disruption [14]. Interestingly, in a seasonal breeder, the mink, the onset of spermatogenesis is not dependent on formation of the impermeable BTB [51]. Therefore, these reports and our VAD study from the prepubertal period to adulthood indicate that assembly of the BTB may not be essential for the initiation and progression of germ cell differentiation in the prepubertal period but may be required for the maintenance of spermatogenesis from the prepubertal period to adulthood.

\section{Acknowledgments}

This work was supported by a Research fellowship from the Japan Society for the Promotion of Science (No. 24002135) and a Grant-in-Aid for Scientific Research B (No. 24380156) from the Ministry of Education, Culture, Sports, Science and Technology of Japan. The research described in this paper was chosen for the Encouragement Award at the 156th Japanese Association of Veterinary Anatomists in Gifu (September 20-22, 2013). We would like to sincerely thank all the people involved.

\section{References}

1. Oakberg EF. A description of spermiogenesis in the mouse and its use in analysis of the cycle of the seminiferous epithelium and germ cell renewal. Am J Anat 1956; 99: 391-413. [Medline]

2. Hogarth CA, Griswold MD. The key role of vitamin A in spermatogenesis. J Clin Invest 2010; 120: 956-962. [Medline]

3. Cheng CY, Mruk DD. Cell junction dynamics in the testis: Sertoli-germ cell interactions and male contraceptive development. Physiol Rev 2002; 82: 825-874. [Medline]

4. Pelletier RM. The blood-testis barrier: the junctional permeability, the proteins and the 
lipids. Prog Histochem Cytochem 2011; 46: 49-127. [Medline]

5. Sun EL, Gondos B. Formation of the blood-testis barrier in the rabbit. Cell Tissue Res 1986; 243: 575-578. [Medline]

6. Morales A, Mohamed F, Cavicchia JC. Apoptosis and blood-testis barrier during the first spermatogenic wave in the pubertal rat. Anat Rec (Hoboken) 2007; 290: 206-214. [Medline]

7. Willems A, Batlouni SR, Esnal A, Swinnen JV, Saunders PT, Sharpe RM, França LR, Gendt K, Verhoeven G. Selective ablation of the androgen receptor in mouse Sertoli cells affects Sertoli cell maturation, barrier formation and cytoskeletal development. PLoS One 2010; 5: e14168. [Medline]

8. Russell L. Movement of spermatocytes from the basal to the adluminal compartment of the rat testis. Am J Anat 1977; 148: 313-328. [Medline]

9. Chihara M, Ostuka S, Ichii O, Hashimoto Y, Kon Y. Molecular dynamics of the bloodtestis barrier components during murine spermatogenesis. Mol Reprod Dev 2010; 77 : 630-639. [Medline]

10. Hasegawa K, Saga Y. Retinoic acid signaling in Sertoli cells regulates organization of the blood-testis barrier through cyclical changes in gene expression. Development 2012; 139: 4347-4355. [Medline]

11. Hellani A, Ji J, Mauduit C, Deschildre C, Tabone E, Benahmed M. Developmental and hormonal regulation of the expression of oligodendrocyte-specific protein/claudin-11 in mouse testis. Endocrinology 2000; 141: 3012-3019. [Medline]

12. Moroi S, Saitou M, Fujimoto K, Sakakibara A, Furuse M, Yoshida O, Tsukita S. Occludin is concentrated at tight junctions of mouse/rat but not human/guinea pig Sertoli cells in testes. Am J Physiol 1998; 274: C1708-C1717. [Medline]

13. Yan HH, Mruk DD, Lee WM, Cheng CY. Blood-testis barrier dynamics are regulated by testosterone and cytokines via their differential effects on the kinetics of protein endocytosis and recycling in Sertoli cells. FASEB J 2008; 22: 1945-1959. [Medline]

14. Gow A, Southwood CM, Li JS, Pariali M, Riordan GP, Brodie SE, Danias J, Bronstein JM, Kachar B, Lazzarini RA. CNS myelin and Sertoli cell tight junction strands are absent in Osp/Claudin-11 null mice. Cell 1999; 99: 649-659. [Medline]

15. Saitou M, Furuse M, Sasaki H, Schulzke JD, Fromm M, Takano H, Noda T, Tsukita S. Complex phenotype of mice lacking occludin, a component of tight junction strands. Mol Biol Cell 2000; 11: 4131-4142. [Medline]

16. Mok KW, Mruk DD, Lee WM, Cheng CY. Spermatogonial stem cells alone are not sufficient to re-initiate spermatogenesis in the rat testis following adjudin-induced infertility. Int J Androl 2012; 35: 86-101. [Medline]

17. Fink C, Weigel R, Fink L, Wilhelm J, Kliesch S, Zeiler M, Bergmann M, Brehm R. Claudin-11 is over-expressed and dislocated from the blood-testis barrier in Sertoli cells associated with testicular intraepithelial neoplasia in men. Histochem Cell Biol 2009; 131: 755-764. [Medline]

18. Baillet A, Mandon-Pepin B. Mammalian ovary differentiation - a focus on female meiosis. Mol Cell Endocrinol 2012; 356: 13-23. [Medline]

19. van Pelt AM, van Dissel-Emiliani FM, Gaemers IC, van der Burg MJ, Tanke HJ, de Rooij DG. Characteristics of A spermatogonia and preleptotene spermatocytes in the vitamin A-deficient rat testis. Biol Reprod 1995; 53: 570-578. [Medline]

20. van Pelt AM, de Rooij DG. Synchronization of the seminiferous epithelium after vitamin A replacement in vitamin A-deficient mice. Biol Reprod 1990; 43: 363-367. [Medline]

21. Ismail N, Morales C, Clermont Y. Role of spermatogonia in the stage-synchronization of the seminiferous epithelium in vitamin A-deficient rats. Am J Anat 1990; 188: 57-63. [Medline]

22. Vernet N, Dennefeld C, Guillou F, Chambon P, Ghyselinck NB, Mark M. Prepubertal testis development relies on retinoic acid but not rexinoid receptors in Sertoli cells. EMBO $J$ 2006; 25: 5816-5825. [Medline]

23. Sugimoto R, Nabeshima Y, Yoshida S. Retinoic acid metabolism links the periodical differentiation of germ cells with the cycle of Sertoli cells in mouse seminiferous epithelium. Mech Dev 2012; 128: 610-624. [Medline]

24. Huang HF, Yang CS, Meyerhofer M, Gould S, Boccabella AV. Disruption of sustentacular (Sertoli) cell tight junctions and regression of spermatogenesis in vitamin A deficient rats. Acta Anat (Basel) 1988; 133: 10-15. [Medline]

25. Morales A, Cavicchia JC. Spermatogenesis and blood-testis barrier in rats after longterm vitamin A deprivation. Tissue Cell 2002; 34: 349-355. [Medline]

26. Ismail N, Morales CR. Effects of vitamin A deficiency on the inter-Sertoli cell tight junctions and on the germ cell population. Microsc Res Tech 1992; 20: 43-49. [Medline]

27. Mark M, Jacobs H, Oulad-Abdelghani M, Dennefeld C, Féret B, Vernet N, Codreanu CA, Chambon P, Ghyselinck B. STRA8-deficient spermatocytes initiate, but fail to complete, meiosis and undergo premature chromosome condensation. J Cell Sci 2008; 121: 3233-3242. [Medline]

28. Zhou Q, Nie R, Li Y, Friel P, Mitchell D, Hess RA, Small C, Griswold MD. Expression of stimulated by retinoic acid gene 8 (Stra8) in spermatogenic cells induced by retinoic acid: an in vivo study in vitamin A-sufficient postnatal murine testes. Biol Reprod 2008; 79: 35-42. [Medline]

29. Hermo L, Pelletier RM, Cyr DG, Smith CE. Surfing the wave, cycle, life history, and genes/proteins expressed by testicular germ cells. Part 1: background to spermatogenesis, spermatogonia, and spermatocytes. Microsc Res Tech 2010; 73: 241-278. [Medline]

30. Hamer G, Gell K, Kouznetsova A, Novak I, Benavente R, Höög C. Characterization of a novel meiosis-specific protein within the central element of the synaptonemal complex. J Cell Sci 2006; 119: 4025-4032. [Medline]

31. La Salle S, Sun F, Zhang XD, Matunis MJ, Handel MA. Developmental control of sumoylation pathway proteins in mouse male germ cells. Dev Biol 2008; 321: 227-237. [Medline]

32. Oka A, Mita A, Takada Y, Koseki H, Shiroishi T. Reproductive isolation in hybrid mice due to spermatogenesis defects at three meiotic stages. Genetics 2010; 186: 339-351. [Medline]

33. Carette D, Weider K, Gilleron J, Giese S, Dompierre J, Bergmann M, Brehm R, Denizot JP, Segretain D, Pointis G. Major involvement of connexin 43 in seminiferous epithelial junction dynamics and male fertility. Dev Biol 2010; 346: 54-67. [Medline]

34. Eid N, Ito Y, Otsuki Y. Enhanced mitophagy in Sertoli cells of ethanol-treated rats: morphological evidence and clinical relevance. J Mol Histol 2012; 43: 71-80. [Medline]

35. Kyrönlahti A, Euler R, Bielinska M, Schoeller EL, Moley KH, Toppari J, Heikinheimo M, Wilson DB. GATA4 regulates Sertoli cell function and fertility in adult male mice. Mol Cell Endocrinol 2011; 333: 85-95. [Medline]

36. Li W, Wu Z, Zhao J, Guo S, Li Z, Feng X, Ma L, Zhang JS, Liu XP, Zhang YQ. Transient protection from heat-stress induced apoptotic stimulation by metastasis-associated protein 1 in pachytene spermatocytes. PLoS One 2011; 6: e26013. [Medline]

37. Russell LD, Ettlin RA, Sinha Hikim AP, Clegg ED. Histological and histopathological evaluation of the testis. Clearwater: Cache River Press; 1990.

38. Dagash H, Mackinnon EA. Testicular microlithiasis: what does it mean clinically? BJU Int 2007; 99: 157-160. [Medline]

39. Ganem JP, Workman KR, Shaban SF. Testicular microlithiasis is associated with testicular pathology. Urology 1999; 53: 209-213. [Medline]

40. Kim B, Winter TC, Ryu J. Testicular microlithiasis: clinical significance and review of the literature. Eur Radiol 2003; 13: 2567-2576. [Medline]

41. Miller FN, Rosairo S, Clarke JL, Sriprasad S, Muir GH, Sidhu PS. Testicular calcification and microlithiasis: association with primary intra-testicular malignancy in 3,477 patients. Eur Radiol 2007; 17: 363-369. [Medline]

42. von Eckardstein S, Tsakmakidis G, Kamischke A, Rolf C, Nieschlag E. Sonographic testicular microlithiasis as an indicator of premalignant conditions in normal and infertile men. J Androl 2001; 22: 818-824. [Medline]

43. Boyer A, Hermo L, Paquet M, Robaire B, Boerboom D. Seminiferous tubule degeneration and infertility in mice with sustained activation of WNT/CTNNB1 signaling in Sertoli cells. Biol Reprod 2008; 79: 475-485. [Medline]

44. O'Shaughnessy PJ, Monteiro A, Verhoeven G, De Gendt K, Abel MH. Occurrence of testicular microlithiasis in androgen insensitive hypogonadal mice. Reprod Biol Endocrinol 2009; 7: 88. [Medline]

45. Anderson EL, Baltus AE, Roepers-Gajadien HL, Hassold TJ, de Rooij DG, van Pelt AM, Page DC. Stra8 and its inducer, retinoic acid, regulate meiotic initiation in both spermatogenesis and oogenesis in mice. Proc Natl Acad Sci USA 2008; 105: 14976-14980. [Medline]

46. Goetz P, Chandley AC, Speed RM. Morphological and temporal sequence of meiotic prophase development at puberty in the male mouse. J Cell Sci 1984; 65: 249-263. [Medline]

47. Chung SS, Sung W, Wang X, Wolgemuth DJ. Retinoic acid receptor alpha is required for synchronization of spermatogenic cycles and its absence results in progressive breakdown of the spermatogenic process. Dev Dyn 2004; 230: 754-766. [Medline]

48. Florin A, Maire M, Bozec A, Hellani A, Chater S, Bars R, Chuzel F, Benahmed M. Androgens and postmeiotic germ cells regulate claudin-11 expression in rat Sertoli cells. Endocrinology 2005; 146: 1532-1540. [Medline]

49. De Gendt K, Swinnen JV, Saunders PT, Schoonjans L, Dewerchin M, Devos A, Tan K, Atanassova N, Claessens F, Lécureuil C, Heyns W, Carmeliet P, Guillou F, Sharpe RM, Verhoeven G. A Sertoli cell-selective knockout of the androgen receptor causes spermatogenic arrest in meiosis. Proc Natl Acad Sci USA 2004; 101: 1327-1332. [Medline]

50. Mazaud-Guittot S, Meugnier E, Pesenti S, Wu X, Vidal H, Gow A, Le MagueresseBattistoni B. Claudin 11 deficiency in mice results in loss of the Sertoli cell epithelial phenotype in the testis. Biol Reprod 2010; 82: 202-213. [Medline]

51. Pelletier RM. Cyclic formation and decay of the blood-testis barrier in the mink (Mustela vison), a seasonal breeder. Am J Anat 1986; 175: 91-117. [Medline] 